\title{
Controlled Diffraction Liquid-Crystal Structures with a Photoalignment Polymer
}

\author{
A. A. Kazak, E. A. Melnikova, A. L. Tolstik*, U. V. Mahilny, and A. I. Stankevich \\ Belarussian State University, Minsk, Belarus \\ *e-mail: tolstik@bsu.by \\ Received March 4, 2008
}

\begin{abstract}
The characteristics of electric-field-controlled diffraction elements based on a nematic liquid crystal and a new photoalignment polymer have been studied. The photocross-linking of this polymer allows periodic oriented structures and liquid crystal phase gratings with a 30\% diffraction efficiency in the first order to be formed.
\end{abstract}

PACS numbers: 42.70.Df, 42.79.Dj, 42.79.Kr, 42.70.Jk

DOI: $10.1134 / \mathrm{S} 1063785008100155$

The unique properties of liquid crystal (LC) elements (small thicknesses of optical layers, low control voltages, high birefringence, etc.) have drawn the attention of researchers and technologists, in particular, in view of broad possibilities offered by LC elements in the creation of data display systems and indicators, light modulators, controllable waveguide structures, and diffraction optics [1-3]. Controllable diffraction elements of various types based on LCs have been developed and different methods of grating formation have been proposed. In early systems [4, 5], the diffraction was controlled using optoelectronic structures of the LC-relief grating type, where the initial LC director orientation was set mechanically, by rubbing a poly(vinyl alcohol) (PVA) layer. Reorientation of the LC director under the action of an applied electric field led to a change in the depth of modulation of the refractive index and, hence, in the diffraction efficiency of the LC grating. An alternative technical solution is based on the use of electrodes of a special shape, which produce a spatially modulated orientation of the LC director [6].

In recent years, successful attempts have been undertaken to orient LCs with the aid of so-called "photoalignment" polymers. According to this approach, a diffraction grating is formed by exposing the polymer to a polarized UV radiation via photolithographic masks or by writing an interference field of two laser beams $[7,8]$. In some cases, an interference pattern can be recorded in the mixture of an LC and a photocrosslinkable polymer without alignment coatings [9].

This study was aimed at creating optoelectronic LC elements with controlled diffraction properties on the basis of periodic alignment structures written by polarized UV radiation in benzaldehyde-containing polymer layers.
We have used polymers containing benzaldehyde fragments as side groups with structures similar to those studied previously $[10,11]$, exhibiting photosensitivity and demonstrating photoinduced birefringence [12]. This anisotropy is stable due to the formation of oriented cross-links between macromolecules under the action of polarized UV radiation [13]. The results presented below demonstrate that an optical anisotropy is induced under the action of such radiation on the surface and in the bulk of indicated polymers, which impart LC-alignment properties to the polymer surface.

Figure 1 shows a schematic diagram of the LC cell with two glass plates 1 bearing transparent indium oxide electrodes 2 . A photosensitive polymer (photopolymer) layer 3 with a thickness of $\sim 0.1 \mu \mathrm{m}$ was applied on the surface of one electrode by centrifuging from a $2 \%$ polymer solution in ethyl acetate. The pho-

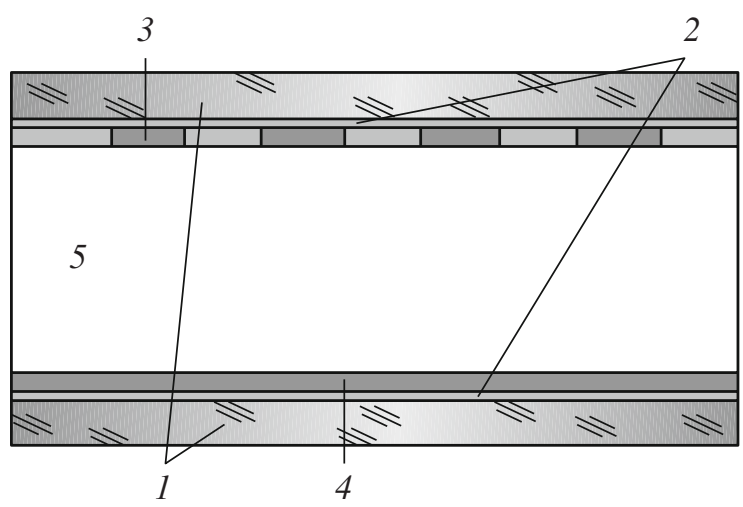

Fig. 1. Schematic diagram of the LC cell: (1) glass plates; (2) transparent indium oxide electrodes; (3) photoalignment polymer layer with a spatially periodic photocross-linked structure; (4) alignment layer (PVA or photopolymer); (5) nematic LC layer. 


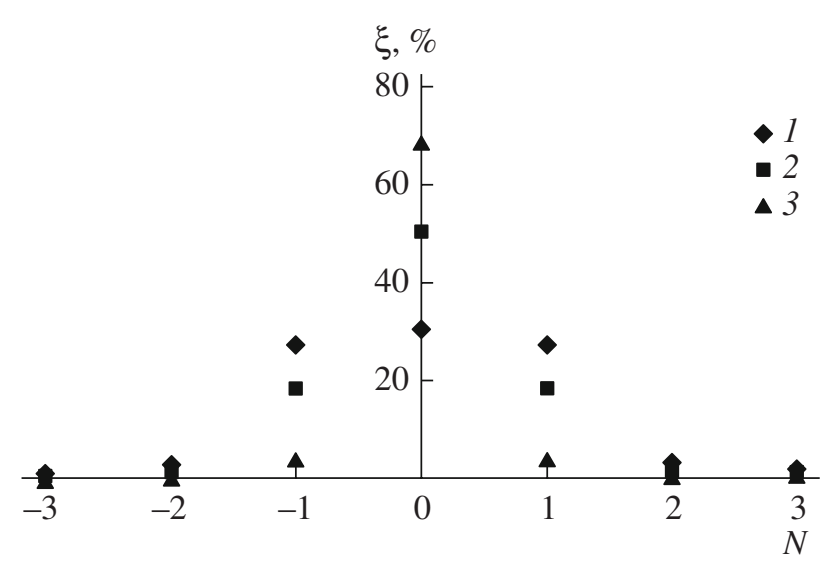

Fig. 2. Plot of the diffraction efficiency $\xi$ in various diffraction orders $N$ for the initial $(1)$ planar and $(2,3)$ twist orientations of the LC layer in the cells manufactured using different materials of the alignment layer: $(1,2)$ PVA; (3) new photoalignment polymer.

topolymer layer was exposed to collimated radiation of a DRSh-250 mercury lamp polarized by reflection from a quartz plate at the Brewster angle. The alignment structures with a period of $80 \mu \mathrm{m}$ were created using a photomask pattern on a quartz substrate. The exposure time was selected so as to ensure a no less than $90 \%$ conversion of the photosensitive component. For this purpose, an analogous film of the same thickness was applied onto a quartz plate and exposed under identical conditions, with the photoconversion monitored using the electronic absorption spectra.

The alignment coating 4 on the second glass plate represented either a thin PVA film or a photopolymer layer uniformly irradiated by polarized light. The assembled optical cell was filled with an LC composition under vacuum to obtain an LC layer with a thickness of $20 \mu \mathrm{m}$. We used a nematic LC 1289 developed at the Research Institute of Organic Intermediate Products and Dyes, Moscow) possessing a birefringence of $n_{\mathrm{e}}-n_{\mathrm{o}}=0.17$. The light beam was incident on the LC cell side with an alignment grating.

We have used three variants of the formation of diffraction LC elements, which corresponded to two directions of the initial orientation (planar alignment and twist structure) and the use of alignment coating 4 with rubbed PVA or a uniformly irradiated photopolymer. The planar alignment appears when the LC director orientation in the exposed photopolymer on one plate coincides with the director orientation on the second plate. The twist structure arises when the LC orientations on the two plates are mutually orthogonal. Figure 2 presents the maximum values of the diffraction efficiency in various orders $(N= \pm 1, \pm 2, \pm 3)$ for the aforementioned variants of the LC director orientation. As can be seen from these data, use of the same alignment polymer (e.g., PVA) for different LC orientations (points 1,2 ) provides the first-order diffraction
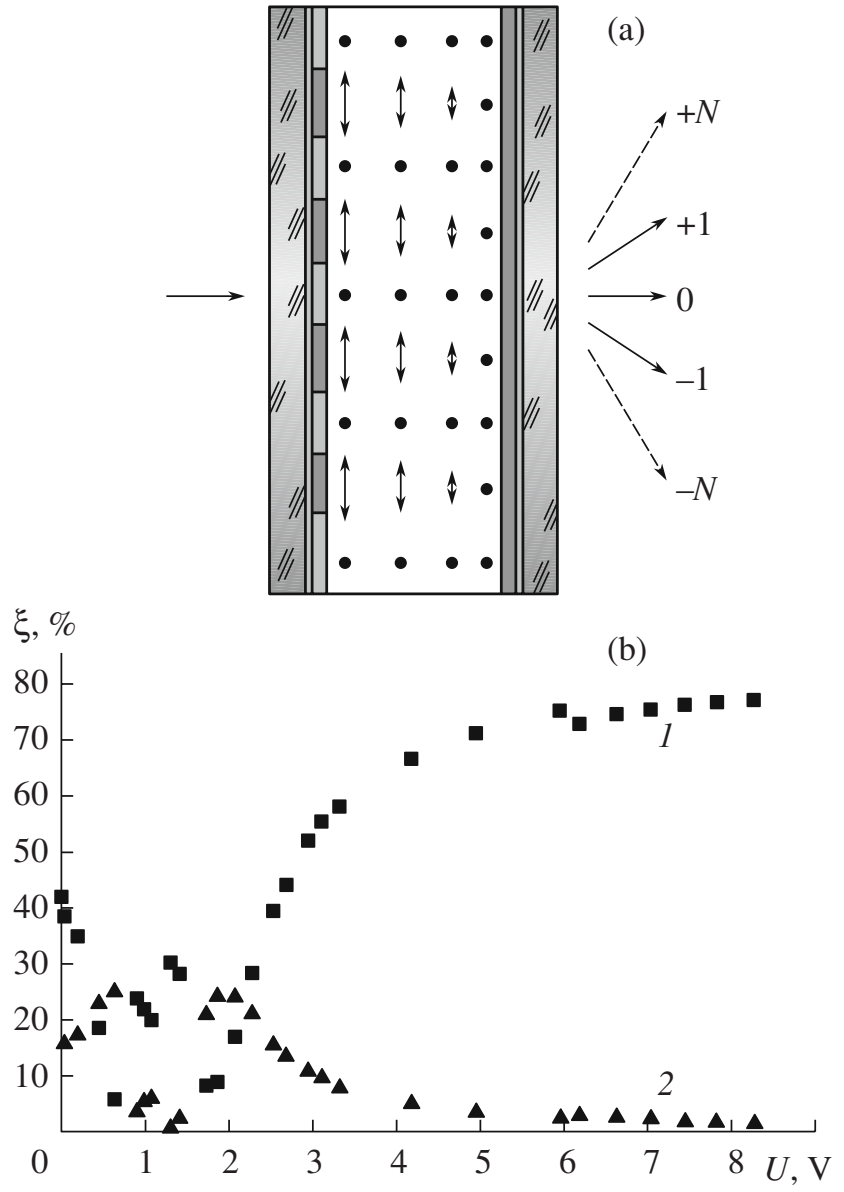

Fig. 3. LC cell with a spatially modulated twist structure: (a) schematic diagram of the cell and diffraction orders; (b) a plot of the diffraction efficiency $\xi$ in the (1) zero order and (2) first versus amplitude $U$ of the applied ac voltage.

efficiency on a level of $20 \%$ for the twist structure and $4 \%$ for the planar orientation. Such a strong dependence of the diffraction efficiency on the LC structure type can be explained by the fact that, for the same director orientation in the exposed photopolymer regions (on the grating lines) and on the second plate, the intermolecular interactions produce partial orientation of the LC molecules in the gaps between lines. In this case, a partly oriented LC structure with a small spatial modulation of the LC directors of layers is formed in volume of the medium. On the other hand, in the case of a twist structure, the competition of two substrates influencing the corresponding alignment polymers leads to the formation of a spatially modulated twist structure in the volume (see Fig. 3a), which accounts for a much greater depth of the refractive index modulation in the LC layer.

We have managed to increase the diffraction efficiency by using a specially developed photopolymer as the alignment layer on the second plate (with a uniform exposure to UV radiation with orthogonal polarization). In this variant of the formation of diffraction LC 
elements, the diffraction efficiency in the first order was about $30 \%$, which is close to the limiting values for thin phase holograms. The diffraction efficiency in the zero order (radiation directly transmitted through the cell) in different variants of LC orientation accordingly decreased (Fig. 2), amounting to 70\% (PVA alignment, planar orientation), 50\% (PVA alignment, twist structure), and 30\% (photopolymer, twist structure).

Control of the diffraction efficiency of optoelectronic LC elements was provided by a bias electric field applied to the cell, which produced reorientation of the LC molecules. In order to avoid screening, we used an ac voltage with a frequency of $1 \mathrm{kHz}$. Figure $3 \mathrm{~b}$ shows a plot of the diffraction efficiency of the LC element versus amplitude of the applied ac voltage. As can be seen from these data, there are optimum values of the control voltage ( $U \approx 0.5$ and $2 \mathrm{~V})$ for which the diffraction efficiency in the first order is maximum. At a zero voltage, the diffraction efficiency is about 1.5 times smaller, which can be explained by the nonsinusoidal profile of the diffraction grating formed for the initial orientation of LC molecules. As the bias field is increased, the orientation of the LC director at the boundaries of lines of the alignment grating is changed so that the grating profile approaches sinusoidal. At a voltage amplitude above $5 \mathrm{~V}$, the LC director changes orientation in the entire layer and a virtually homogeneous homeotropic structure is formed, in which a light beam propagates predominantly along the axis of the birefringent crystal and the diffraction efficiency is small.

The existence of two optimum values of the applied voltage is related to the features of light diffraction on anisotropic gratings with a difference between the optical lengths for ordinary and extraordinary waves that is several times greater compared to the laser wavelength. This situation was theoretically analyzed, for example, in [14] where oscillating dependences of the diffraction efficiency on the microrelief depth in an anisotropic grating were obtained for various polarizations of the incident wave.

In conclusion, we demonstrated the possibility of electrical control of the diffraction efficiency of an LC structure employing a new photoalignment poly- mer. Optimum conditions for the formation of diffraction gratings in the LC layer have been established and the working range of control bias voltage amplitudes has been determined.

\section{REFERENCES}

1. T. Matsui, M. Ozaki, and K. Yoshino, J. Opt. Soc. Am. B 21, 1651 (2004).

2. P. Pagliusi and G. Gipparrone, J. Opt. Soc. Am. B 21, 996 (2004).

3. T.-H. Lin and A.Y.-G. Fuh, Opt. Lett. 30, 1390 (2005).

4. V. M. Durasov, A. S. Rubanov, I. V. Stashkevich, and A. V. Chalei, Zh. Prikl. Spektr. 47 (3), 19 (1987).

5. A. S. Rubanov, I. V. Stashkevich, A. V. Chalei, and V. M. Durasov, USSR Inventor's Certificate no. 134775 (Novemer 26, 1985).

6. M. Bouvier and T. Scharf, Opt. Eng. 39, 2129 (2000).

7. H. Sarkissian, S. V. Serak, N. V. Tabiryan, et al., Opt. Lett. 31, 2248 (2006).

8. V. Kapoustine, A. Kazakevitch, V. So, and R. Tam, Opt. Commun. 266, 1 (2006).

9. J. Zhou and D. M. Collard, Opt. Lett. 31, 652 (2006).

10. I. P. Zyat'kov and V. V. Mogil'nyi, Zh. Prikl. Spektr. 41, 466 (1984) [J. Appl. Spectrosc. 41, 1060 (1984)].

11. V. V. Mogil'ny̌̆, and A. I. Stankevich, Zh. Nauchn. Prikl. Fotogr. Kinematogr. 31, 407 (1986).

12. V. V. Mogil'ny $\breve{1}$, and A. I. Stankevich, in Low-Dimensional Systems 2: Physial Chemistry of Elementov and Systems with Low-Dimensional Structures (Synthesis, Diagnostic, and Applicationh of New Materials and Structures). A Collection of Scientific Papers, Ed. by S. A. Maskevich et al. (Grodno State University, Grodno, 2003), p. 134 [in Russian].

13. V. V. Mogil'ny̌̆, and A. I. Stankevich, Proceedings of the 5th Int. Conf. on the Intenraction of Radiation with Solids (Minsk, 2003), Part 1, p. 179.

14. V. V. Belyaev, E. M. Kushnir, A. V. Klyshkov, and V. I. Tsoi, Opt. Zh. 72 (9), 86 (2005) [J. Opt. Technol. 72, 725 (2005)].

Translated by P. Pozdeev 